\title{
A GENERALISATION OF THE STUDY OF SUM AND SQUARE LAW SIGNAL PROCESSORS WITH MULTIPLE CLIPPED INPUTS
}

\author{
R. G. KEATS and V.K-K. YU
}

(Received 28 January 1976)

(Revised 26 July 1976)

\begin{abstract}
The recent work of Cheng and Stokes on the processing of clipped signals from two or three receivers is extended and generalised by removing a number of restrictions. In particular, there is no restriction on the number of receivers and the restrictions on the statistical properties of the signal and noise processes have been considerably relaxed.

Mathematically - Plackett's result is used to expand the orthant probabilities involved in increasing powers of the input signal to noise ratio.
\end{abstract}

\section{Introduction}

The application of statistical hypothesis testing to the detection of signals in additive background noise is well established, see, for example, Helstrom [8], Van Trees [21], and more recent work by Kailath [9], [10], [11]. In many of these applications the outputs of a number of receivers are combined and processed to give a quantity on which a decision concerning the presence or absence of a signal may be based. This paper is concerned with sum and square law processors as illustrated in Figure (1), and in particular, a comparison is made of the performance of two such processors; viz., the analogue sum and square law processor (A.S.S.P.), and the polarity sum and square law processor (P.S.S.P.). This latter processor (P.S.S.P.) has several attractive features in practical realisations, notably a considerable reduction in the amount of computing equipment required, and there is, therefore, continuing interest in its comparative performance. Similar signal processors involving clipping will also be of interest for many years, since much new equipment presently being designed incorporates such processing, e.g. [1]. 
Early work in this field was described by Faran and Hills [7] and other authors; for example, [6], [12], [17], [19], [20]. This work was continued by Cheng [3] and both he and Stokes give extensive resumés of this and related work in their theses, Cheng [2] and Stokes [18]. The approach in this paper was first presented to the Australian Conference on Signal Processing for Arrays, Keats [13].

The measure, used by many authors, of the performance of processors similar to those discussed in this paper, is the ratio of signal power to noise power at the output of the processor. The signal power is defined as the square of the difference between the mean output in the presence of signal and the mean output in the absence of signal. The noise power is defined as the variance of the output in the presence of signal [6], [7], [19]. This choice of signal to noise ratio, in particular the definition of noise power, leads to an interesting mathematical problem, which the authors believe has not been seriously tackled before. A new and unexpected result, which is discussed in section 4 and appendix 3, arises directly from this choice. Other measures of performance have also been used by various authors, in particular the signal to noise ratio as above but with the noise power defined as the variance of the output in the absence of signal [5]. Although this definition of signal to noise ratio has as much justification as the one used in this paper, it does not lead to any unexpected or new results.

The operation of the class of processors discussed here is shown in Figure (1). The inputs to each receiver, $R_{t}$, consist of a signal $S$ plus noise $N_{t}$; the subscript $i$ indicates that the noise inputs at each receiver may differ, whereas the signal input $S$ is the same at each receiver. These inputs are assumed to be realisations of stationary random processes. Although the signals at each receiver are identical realisations of the same process, it is possible that they are shifted in time relative to each other. It will be assumed that this shift is not present, or, alternatively, delays are inserted immediately following each receiver to synchronize the signals from all receivers. Following the time delay the input to each receiver may be transformed linearly or non-linearly; the members of the class of processors studied here differ only in the type of transformation used at this point. In the case of the A.S.S.P. the transformation is the identity; whereas the P.S.S.P. incorporates the transformation

$$
A_{1}=\operatorname{sgn}\left[I_{1}\right]
$$

where $I_{i}=S+N_{i}$, and the function sgn is defined by

$$
\begin{array}{ll}
\operatorname{sgn}(x)=1, & x \geqq 0, \\
\operatorname{sgn}(x)=-1, & x<0 .
\end{array}
$$




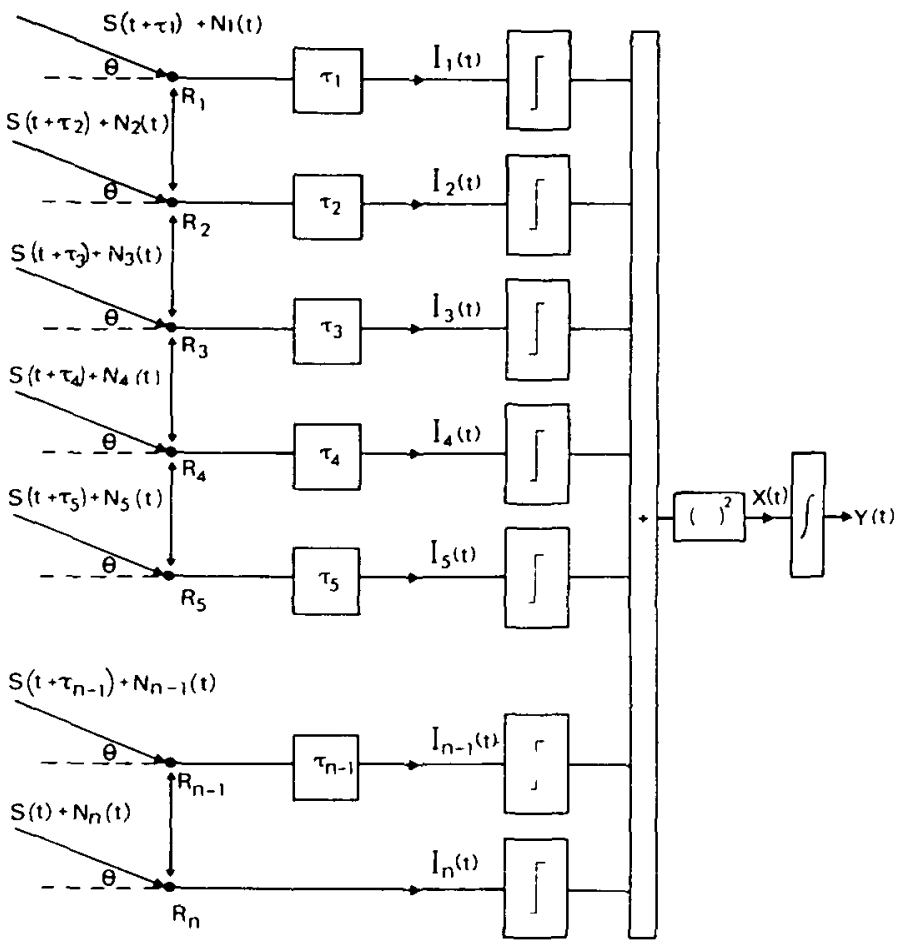

Figure 1 - A sum and square law processor

Although one of the two cases compared in this paper uses the identity transformation, any linear transformation may be studied similarly and with very little added complication, since the relation between the statistics of a Gaussian input to a linear filter and its output is well known; see, for example, Laning and Battin [14].

Following these transformations, the outputs $A_{t}$ are summed and squared to give the quantity $X=\left[\Sigma A_{6}\right]^{2}$ which is then integrated, using the weighting function

$$
W(t)=\frac{1}{T} \exp \left(\frac{-t}{T}\right)
$$

to give the processor output

$$
Y(t)=\frac{1}{T} \int_{0}^{\infty} e^{-\mu / T} X(t-\mu) d \mu .
$$

The following additional assumptions will be made. 
(a) The signal $S(t)$ and the noise $N_{i}(t)$ present at each receiver are stationary normal random processes with zero mean, variances $\sigma_{s}^{2}, \sigma_{\imath}^{2}$, and normalized correlation functions $\rho_{s}(\tau)$ for the signal and $\rho_{i}(\tau)$ for each $N_{1}(t)$.

(b) All cross-correlation functions are identically zero.

Stokes [18], following the example of earlier authors [3], [7], considered the special case in which the noise variances were all equal and the normalized correlation functions of both signal and noise were identical. The method used in this paper generalises the problem considerably, but does involve the further assumption:

(c) the input signal to noise ratio is small.

This last assumption is not restrictive in most, if not all, practical cases.

\section{Previous relevant results}

The following results will be used in this paper without derivation. They have been quoted and used by many authors and can all be found in Stokes [18]; some, as indicated can be found in more readily available publications. Denoting the number of receivers by $n$,

$$
\begin{aligned}
& E[Y]=E[X]=E\left(\sum_{i=1}^{n} A_{i}\right)^{2}=\sum_{i=1}^{n} E\left[A_{i}^{2}\right]+\sum_{i \neq 1} E\left[A_{i} A_{i}\right] \\
& \sigma_{Y}^{2}=\frac{1}{T} \int_{0}^{\infty} e^{-\mu / T} \rho_{X}(\mu) d \mu, \quad[6] ;[7],
\end{aligned}
$$

where: $\rho_{X}(\mu)$ is the covariance function of $X$.

If we define $B_{1}$ to be the quantity $A_{1}$ delayed by $\mu$, then

$$
\begin{aligned}
\rho_{X}(\mu)= & E\left[\left(\Sigma A_{l}\right)^{2}\left(\Sigma B_{l}\right)^{2}\right]-E^{2}\left(\Sigma A_{l}\right)^{2} \\
= & E\left[\left(\Sigma A_{l}^{2}\right)\left(\Sigma B_{l}^{2}\right)\right]+2 E\left[\left(\Sigma A_{l}^{2}\right)\left(\Sigma^{\prime} B_{l} B_{l}\right)\right] \\
& +2 E\left[\left(\Sigma B_{l}^{2}\right)\left(\Sigma^{\prime} A_{l} A_{l}\right)\right]+4 E\left[\Sigma^{\prime} A_{l} A_{l} B_{l} B_{l}\right] \\
& +4 E\left[\Sigma^{\prime} A_{l} A_{l} B_{l} B_{k}\right]+4 E\left[\Sigma^{\prime} A_{\imath} A_{l} B_{k} B_{l}\right]-E^{2}\left[\Sigma A_{l}^{2}+2 \Sigma^{\prime} A_{l} A_{l}\right],
\end{aligned}
$$

where $\Sigma^{\prime}$ indicates that the summation is to be taken over all terms such that $i<j, k<l$ and all distinct indices take different values.

The nub of the problem thus becomes the calculation of this covariance function $\rho_{x}(\mu)$.

In the case of the A.S.S.P. the following results are fairly trivial extensions of those appearing in the literature [7], [18]. For this case the following results apply.

(a) In the absence of signal, 


$$
E[Y(t)]=E[X(t)]=\sum_{i=1}^{n} \sigma_{t}^{2}
$$

(b) In the presence of signal,

$$
E[Y(t)]=E[X(t)]=n^{2} \sigma_{s}^{2}+\sum_{i=1}^{n} \sigma_{1}^{2},
$$

while the autocorrelation function

$$
\rho_{X}(\tau)=2\left[n^{2} \sigma_{s}^{2} \rho_{s}(\tau)+\sum_{i=1}^{n} \sigma_{i}^{2} \rho_{i}(\tau)\right]^{2}
$$

From these relations the signal to noise ratio at the output of this processor may be found to be

$$
\operatorname{SNR}_{\mathrm{ASSP}(n)}=\frac{n^{4} \sigma_{S}^{4}}{\frac{2}{T} \int_{0}^{\infty} \exp \left(-\frac{\mu}{T}\right)\left(\sum K_{1}(\mu)\right)^{2} d \mu},
$$

where:

$$
K_{\imath}(\mu)=n \sigma_{s}^{2} \rho_{s}(\mu)+\sigma_{\imath}^{2} \rho_{i}(\mu) .
$$

In the case considered by several authors; e.g. [2], [7], [18], viz.,

$$
\rho_{s}(\mu)=\rho_{i}(\mu)=\rho(\mu), \text { all } i,
$$

and

$$
\sigma_{\imath}^{2}=\sigma_{N}^{2}, \quad \text { all } i
$$

the signal to noise ratio for this processor becomes

$$
\frac{n^{2} a^{2}}{2 K[1+(n-1) a]^{2}}
$$

where:

$$
a=\frac{\sigma_{S}^{2}}{\sigma_{S}^{2}+\sigma_{N}^{2}}
$$

and

$$
K=\frac{1}{T} \int_{0}^{\infty} \exp \left(-\frac{\mu}{T}\right) \rho^{2}(\mu) d \mu .
$$

Cheng [2] has solved the problem of the P.S.S.P. in the case of two inputs and the assumptions (5) and (6). His result can be written

$$
\mathrm{SNR}_{\mathrm{PSSP}(2)}=\frac{(\arcsin a)^{2}}{\frac{\pi^{2}}{16 T} \int_{0}^{\infty} \exp \left(-\frac{\mu}{T}\right) \rho_{X}(\mu) d \mu}
$$


where:

$$
\rho_{X}(\mu)=\frac{16}{\pi^{2}}\left\{[\arcsin \rho(\mu)]^{2}-[\arcsin a \rho(\mu)]^{2}\right\}
$$

Stokes endeavoured to extend Cheng's results to $n=3$ but was unable to find a closed form expression for the term $E\left[\Sigma^{\prime} A_{t} A_{1} B_{i} B_{k}\right]$ in $\rho_{X}(\mu),(3)$; he obtained numerical values by a most complicated computational programme. This difficulty again arose when he endeavoured to extend the problem to higher values of $n$, but as can be seen from (3) modified by the assumptions (5) and (6) no further difficulties arise. In fact, Stokes' expression for $\rho_{X}(\mu)[18, p$. 152] becomes

$$
\begin{aligned}
& 2 n(n-1) E\left[A_{i} A_{l} B_{i} B_{l}\right]+4 n(n-1)(n-2) E\left[A_{l} A_{j} B_{j} B_{k}\right] \\
& +n(n-1)(n-2)(n-3) E\left[A_{i} A_{i} B_{k} B_{l}\right]-\frac{4}{\pi^{2}} n^{2}(n-1)^{2}[\arcsin a]^{2}
\end{aligned}
$$

Closed form expressions are known [4] in this case for $E\left[A_{i} A_{j} B_{1} B_{1}\right]$ and $E\left[A_{1} A_{1} B_{k} B_{l}\right]$, but after a long search no closed form could be found for $E\left[A, A, B, B_{k}\right]$.

These three expectations all depend on the evaluation of orthant quadrivariate probabilities; i.e., the evaluation of fourfold integrals of the form

$$
\left(\frac{1}{2 \pi}\right)^{2}|Q|^{\frac{1}{2}} \int_{0}^{\infty} \int_{0}^{\infty} \int_{0}^{\infty} \int_{0}^{\infty} \exp -\frac{1}{2}\left(x^{T} Q x\right) d x
$$

where $Q=R^{-1}$ and $R$ is the correlation matrix of four jointly normal variates having zero mean and unit variance. For the three expectations above, the matrix $R$ has the form

$$
\begin{aligned}
& {\left[\begin{array}{cccc}
1 & a & b & -a b \\
a & 1 & a b & -b \\
b & a b & 1 & -a \\
-a b & -b & -a & 1
\end{array}\right],} \\
& {\left[\begin{array}{cccc}
1 & a & a b & a b \\
a & 1 & b & a b \\
a b & b & 1 & a \\
a b & a b & a & 1
\end{array}\right],}
\end{aligned}
$$

and 


$$
\left[\begin{array}{cccc}
1 & a & a b & -a b \\
a & 1 & a b & -a b \\
a b & a b & 1 & -a \\
-a b & -a b & -a & 1
\end{array}\right] .
$$

The one involving matrix (11) proved intractable.

\section{Output signal to noise ratio of the P.S.S.P. ${ }_{(n)}$}

Progress in the general P.S.S.P. problem became possible when attempts to obtain a closed form expression for all the relevant orthant probabilities were abandoned and a new approach adopted [13]. Since the cases of practical interest were confined to those where the signal to noise ratio, and therefore the parameter $a$, were small, it was decided to look for a series expansion of expressions like (9). In this general case the matrices have five parameters and are, for example, of the form

$$
\left[\begin{array}{cccc}
1 & a & a b c+d & a b \\
a & 1 & a b & \frac{a b}{c}+e \\
a b c+d & a b & 1 & a \\
a b & \frac{a b}{c}+e & a & 1
\end{array}\right] .
$$

To avoid confusion in notation, we will take advantage of the fact that correlation matrices are symmetric and write the general orthant probability for four variates as

$$
F\left(\rho_{12}, \rho_{13}, \rho_{14}, \rho_{23}, \rho_{24}, \rho_{34}\right),
$$

i.e., $F$ is a function of the six correlation coefficients of the four variates involved. In particular cases we may also use notations such as $\Phi(a, b, c, d, e)$ so that in the case (13)

$$
F\left(a, a b c+d, a b, a b, \frac{a b}{c}+e, a\right)=\Phi(a, b, c, d, e) .
$$

We will also use the notation $\Phi, \Phi^{\prime}, \Phi^{\prime \prime}, \cdots, \Phi^{(m)}$ to represent the function $\Phi(a, b, c, d, e)$ or its partial derivatives evaluated at $a=0$.

Expanding $\Phi(a, b, c, d, e)$ at $a=0$

$$
\Phi(a, b, c, d, e)=\Phi+a \Phi^{\prime}+\frac{a^{2}}{2 !} \Phi^{\prime \prime}+\cdots
$$

and the problem reduces to evaluating the expressions $\Phi, \Phi^{\prime}$, etc. 
Plackett's result [16] may be applied to the evaluation of these expressions, since in general

$$
\Phi^{\prime}=\left(\sum \frac{\partial F}{\partial \rho_{i j}} \frac{\partial \rho_{i j}}{\partial a}\right)_{a=0}
$$

with more complicated but still manageable expressions for higher derivatives. Expressions for $\partial \rho_{t l} / \partial a$ are trivial while expressions such as $\partial^{2} F / \partial \rho_{l} \partial \rho_{k l}$ follow from [16].

For example, following Plackett [16], we have

$$
\frac{\partial^{2} F}{\partial \rho_{1,} \partial \rho_{k I}}=\left(\frac{1}{2 \pi}\right)^{2}|Q|^{\frac{1}{2}} \int_{0}^{\infty} \int_{0}^{\infty} \int_{0}^{\infty} \int_{0}^{\infty} \frac{\partial}{\partial x_{1}} \frac{\partial}{\partial x_{1}} \frac{\partial}{\partial x_{k}} \frac{\partial}{\partial x_{l}} \exp -\frac{1}{2}\left(x^{T} Q x\right) d x
$$

and the value of this expression at $a=0$ is easily obtained in the following way. Since $a$ is not involved in either the integrations or the differentiations we are only concerned with the matrix $Q_{0}$; i.e., $Q$ evaluated at $a=0$; e.g., in the example (13).

$$
Q_{0}^{-1}=\left[\begin{array}{llll}
1 & 0 & d & 0 \\
0 & 1 & 0 & e \\
d & 0 & 1 & 0 \\
0 & e & 0 & 1
\end{array}\right]
$$

Furthermore, if all the indices $i, j, k, l$ are distinct it is easily seen that

$$
\begin{aligned}
\frac{\partial^{2} \Phi}{\partial \rho_{l l} \partial \rho_{k l}} & =\left(\frac{1}{2 \pi}\right)^{2}\left|Q_{o l}\right|^{\frac{1}{2}} \\
& =\frac{1}{4 \pi^{2} \sqrt{\left(1-d^{2}\right)\left(1-e^{2}\right)}} .
\end{aligned}
$$

Similarly if $i=k=1, j=l=2$, we have

$$
\begin{gathered}
\left(\frac{\partial^{2} \Phi}{\partial \rho_{12}^{2}}\right)_{a \rightarrow(1)}=\left(\frac{1}{2 \pi}\right)^{2}\left|Q_{0}\right|^{\frac{1}{2}} \int_{11}^{\infty} \int_{0}^{\infty} \int_{10}^{\infty} \int_{0}^{\infty}\left(\frac{\partial}{\partial x_{1}}\right)^{2}\left(\frac{\partial}{\partial x_{2}}\right)^{2} \exp -\frac{1}{2}\left(x^{T} Q_{0} x\right) d x \\
Q_{00}=\left[\begin{array}{cccc}
\frac{1}{1-d^{2}} & 0 & \frac{-d}{1-d^{2}} & 0 \\
0 & \frac{1}{1-e^{2}} & 0 & \frac{-e}{1-e^{2}} \\
\frac{-d}{1-d^{2}} & 0 & \frac{1}{1-d^{2}} & 0 \\
0 & \frac{-e}{1-e^{2}} & 0 & \frac{1}{1-e^{2}}
\end{array}\right] .
\end{gathered}
$$


So that

$$
\begin{aligned}
\left(\frac{\partial^{2} \Phi}{\partial \rho_{12}^{2}}\right)_{a=0}= & \left(\frac{1}{2 \pi}\right)^{2}\left|Q_{0}\right|^{\frac{1}{2}} \int_{0}^{\infty} \int_{0}^{\infty} \frac{e d}{\left(1-d^{2}\right)\left(1-e^{2}\right)} x_{3} x_{4} \\
& \times \exp -\frac{1}{2}\left(\frac{x_{3}^{2}}{1-d^{2}}+\frac{x_{4}^{2}}{1-e^{2}}\right) d x_{3} d x_{4} \\
= & \left(\frac{1}{2 \pi}\right)^{2} \frac{e d}{\sqrt{\left(1-d^{2}\right)\left(1-e^{2}\right)}}
\end{aligned}
$$

Returning to the general P.S.S.P.(n) we wish to determine expressions for the terms involved in $\rho_{X}(\mu)$. In this case equation (3) becomes

$$
\begin{aligned}
& n^{2}+2 n E\left[\Sigma^{\prime} A_{l} A_{\jmath}+B_{l} B_{l}\right]+4 E\left[\Sigma^{\prime} A_{l} A_{l} B_{l} B_{l}\right] \\
& +4 E\left[\Sigma^{\prime} A_{1} A_{1} B_{1} B_{k}\right]+4 E\left[\Sigma^{\prime} A_{1} A_{1} B_{k} B_{l}\right]-\left(n+2 E\left[\Sigma^{\prime} A_{1} A_{l}\right]\right)^{2} \text {. }
\end{aligned}
$$

Using the result

$$
\begin{aligned}
& E\left[A_{1} A_{j}\right]=E\left[B_{1} B_{l}\right]=\frac{2}{\pi} \arcsin a_{i,}, \\
& a_{i j}=\frac{\sigma_{S}^{2}}{\sqrt{\left(\sigma_{S}^{2}+\sigma_{l}^{2}\right)\left(\sigma_{S}^{2}+\sigma_{l}^{2}\right)}}
\end{aligned}
$$

we obtain the following expression for $\rho_{X}(\mu)$ :

$$
4 E\left[\Sigma^{\prime} A_{t} A_{1} B_{\imath} B_{1}+\Sigma^{\prime} A_{\imath} A_{1} B_{l} B_{k}+\Sigma^{\prime} A_{t} A_{1} B_{k} B_{l}\right]-\frac{4}{\pi^{2}}\left(\Sigma^{\prime} 2 \arcsin a_{\imath}\right)^{2}
$$

Accordingly, we require expressions for the three quantities $E\left[A_{1} A_{1} B_{1} B_{1}\right]$, $E\left[A, A, B, B_{k}\right]$ and $E\left[A, A, B_{k} B_{l}\right]$. These expectations may be written in terms of orthant probabilities as below, where the following notation in addition to (17) has been introduced:

$$
\begin{aligned}
& b=\rho_{S}(\mu), \quad c_{i j}=\left(\frac{\sigma_{S}^{2}+\sigma_{i}^{2}}{\sigma_{S}^{2}+\sigma_{j}^{2}}\right)^{1 / 2}, \quad d_{i}=\frac{\sigma_{i}^{2} \rho_{i}(\mu)}{\sigma_{S}^{2}+\sigma_{l}^{2}} . \\
& E\left[A_{i} A, B_{i} B_{l}\right]=2 P\left[A_{i} A_{1} B_{i} B_{j}=1\right]-1 \\
& =4\left[\Phi_{1}\left(a_{i j}, b, c_{l}, d_{i}, d_{l}\right)+\Phi_{1}\left(a_{i j},-b, c_{i j},-d_{i},-d_{l}\right)\right. \\
& \left.\quad+\Phi_{1}\left(-a_{i j}, b,-c_{p}, d_{i}, d_{l}\right)+\Phi_{1}\left(-a_{i j},-b,-c_{j},-d_{i},-d_{l}\right)\right]-1
\end{aligned}
$$

where:

$$
\Phi_{1}(a, b, c, d, e)=F\left[a, a b c+d, a b, a b, \frac{a b}{c}+e, a\right]
$$

and therefore 


$$
\Phi_{1}(0, b, c, d, e)=F[0, d, 0,0, e, 0] .
$$

Similarly $E\left[A_{1} A_{j} B, B_{k}\right]$ is given by

$$
\begin{aligned}
& 4\left[\Phi_{2}\left(a_{i j}, b, c_{j k}, c_{i j}, d_{1}\right)+\Phi_{2}\left(a_{i j},-b, c_{j k}, c_{i j},-d_{j}\right)\right. \\
& \left.\quad+\Phi_{2}\left(-a_{i j},-b,-c_{j k},-c_{i j},-d_{j}\right)+\Phi_{2}\left(-a_{i j}, b,-c_{j k},-c_{i j}, d_{1}\right)\right]-1
\end{aligned}
$$

where:

$$
\Phi_{2}(a, b, c, d, e)=F[a, a b, a b c, a b d+e, a b c d, a c d],
$$

and therefore

$$
\Phi_{2}(0, b, c, d, e)=F[0,0,0, e, 0,0]
$$

Finally

$$
\begin{aligned}
E[ & \left.A_{i} A_{j} B_{k} B_{l}\right] \\
= & 4\left[\Phi_{3}\left(a_{i \jmath}, b, c_{j k}, c_{j l}, c_{i k}\right)+\Phi_{3}\left(a_{i l},-b, c_{l k}, c_{l l}, c_{i k}\right)\right. \\
& \left.+\Phi_{3}\left(-a_{i l}, b,-c_{j k}, c_{l l}, c_{i k}\right)+\Phi_{3}\left(-a_{i j},-b,-c_{l k}, c_{i l}, c_{i k}\right)\right]-1
\end{aligned}
$$

where:

$$
\begin{aligned}
& \Phi_{3}(a, b, c, d, e)=F\left[a, a b c, a b d, a b e, \frac{a b d e}{c}, a d e\right], \\
& \Phi_{3}(0, b, c, d, e)=F[0,0,0,0,0,0] .
\end{aligned}
$$

It is the simple form of the expressions (20), (22) and (24) which makes the calculation of the required partial derivatives a practical proposition.

It is easily seen that:

$$
\begin{aligned}
& \Phi_{1}\left(0, b, c_{j}, d_{i}, d_{l}\right)=\frac{1}{4 \pi^{2}} \arccos \left(-d_{i}\right) \arccos \left(-d_{i}\right), \\
& \Phi_{2}\left(0, b, c_{j k}, c_{i j}, d_{l}\right)=\frac{1}{8 \pi} \arccos \left(-d_{l}\right)
\end{aligned}
$$

and

$$
\Phi_{3}\left(0, b, c_{j k}, c_{l l}, c_{i k}\right)=\frac{1}{16}
$$

Appendix (1) contains the first three partial derivatives required for the evaluation of $\Phi^{\prime}, \Phi^{\prime \prime}$ and $\Phi^{\prime \prime \prime}$ and the required expectations follow in Appendix (2).

In order to evaluate the output signal to noise ratio for the P.S.S.P. ${ }_{(n)}$ it remains to determine: 
(a) the square of the difference between the mean output in the presence of signal and the mean output in the absence of signal,

(b) $\frac{1}{T} \int_{0}^{\infty} \exp \left(-\frac{\mu}{T}\right) \rho_{x}(\mu) d \mu$.

The first of these quantities is easily seen to be $\left[\left(n+(4 / \pi) \Sigma^{\prime} \arcsin a_{u \prime}\right)-n\right]^{2}$ while the second may be found by substituting the appropriate expectations in the expression (18) for $\rho_{X}(\mu)$ and carrying out the single integration, numerically if necessary.

\section{Discussion and an example}

The work reported here describes a general method of computing the output signal to noise ratio of the P.S.S.P.(n) and comparing it with the same ratio for the A.S.S.P.(n). The assumptions (a), (b), (c) listed in section 1 are not restrictive and will cover many practical examples. The problem could be further generalised by removing assumption (b), but the matrices $Q_{0}$ would then no longer have the simple forms given in section 3 .

As an example we will consider the less complicated problem studied by Stokes [18]. The following restrictions are introduced solely to reduce the amount of calculation involved. There is no additional difficulty when all parameters have different values. Accordingly, it will be assumed:

$$
\begin{gathered}
\sigma_{\imath}^{2}=\sigma_{N}^{2}, \quad \text { all } i, \\
\rho_{s}(\mu)=\rho_{\imath}(\mu)=\rho(\mu), \quad \text { all } i .
\end{gathered}
$$

Under these circumstances the signal to noise ratio of the A.S.S.P.(n) becomes

$$
\frac{n^{2} a^{2}}{2 K[1+(n-1) a]^{2}}
$$

as given in section 2 .

The signal to noise ratio of the P.S.S.P.(n) after neglecting terms of order $a^{4}$ and higher may be written in the form

where:

$$
\frac{n(n-1) a^{2}}{\alpha n^{2}+\beta n+\gamma}
$$

$$
\begin{aligned}
& \alpha=2 a^{2} K(1-2 a), \\
& \beta=4 a I_{1}+a^{2}\left(4 I_{3}-10 K-4\right)+a^{3}\left(\frac{2}{3} I_{2}-4 I_{4}+20 K\right), \\
& \gamma=2 I_{5}-8 a I_{1}+a^{2}\left(10 K-8 I_{3}+8\right)+a^{3}\left(8 I_{4}-\frac{4}{3} I_{2}-24 K\right) ;
\end{aligned}
$$

the quantity $K$ was defined in section 2 . 
The integrals $I_{1}$ to $I_{5}$ are of the form

$$
\begin{aligned}
& I_{1}=\frac{1}{T} \int_{0}^{\infty} \exp \left(-\frac{\mu}{T}\right) b \arcsin (b) d \mu, \\
& I_{2}=\frac{1}{T} \int_{0}^{\infty} \exp \left(-\frac{\mu}{T}\right) b^{3} \arcsin (b) d \mu, \\
& I_{3}=\frac{1}{T} \int_{0}^{\infty} \exp \left(-\frac{\mu}{T}\right)\left(1-b^{2}\right)^{\frac{1}{2}} d \mu, \\
& I_{4}=\frac{1}{T} \int_{0}^{\infty} \exp \left(-\frac{\mu}{T}\right)\left(1-b^{2}\right)^{\frac{1}{2}} b^{2} d \mu, \\
& I_{5}=\frac{1}{T} \int_{0}^{\infty} \exp \left(-\frac{\mu}{T}\right)[\arcsin (b)]^{2} d \mu .
\end{aligned}
$$

All these integrals may be evaluated once the correlation function $b=\rho(\mu)$ is given.

Three separate cases with corresponding approximations for (26) may be recognised. For $n$ large, say $n>10$, we have

Case $\quad$ S.N.R.P.S.S.P. ${ }_{(n)}$

$$
\begin{array}{ll}
n a \ll 1 & \frac{n^{2} a^{2}}{2 I_{5}} \\
n a \simeq 1 & \frac{n^{2} a^{2}}{2 K n^{2} a^{2}+4 I_{1} n a+2 I_{5}} \\
n a \gg 1 & \frac{1}{2 K(1-2 a)}
\end{array}
$$

The expression (26) has been evaluated for a range of values of $n$ using the parameter values,

$$
a=\frac{1}{16}, \quad b=\rho(\mu)=e^{-2|\mu|},
$$

and for $T=$ both 5 and 100. The results appear in logarithmic form in Figures (2) and (3). The quotient of the signal to noise ratio of the A.S.S.P. ${ }_{(n)}$ divided by that of the P.S.S.P. ${ }_{(n)}$ appears in Figure (4); the graph for $T=5$ has been reproduced here; that for $T=100$ is very little different.

As would be expected, the performance of each processor improves as 


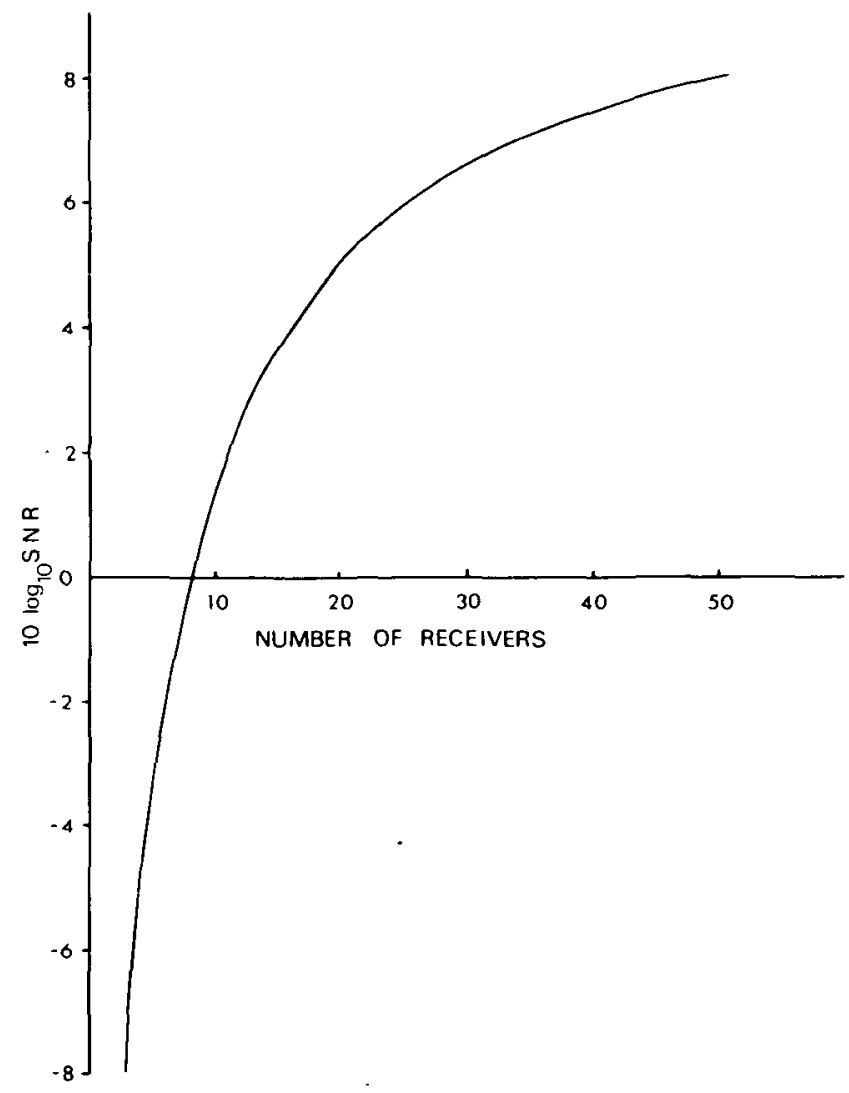

Figure 2-Output signal to noise ratio of the polarity sum and square law processor for $T=5$

the number of receivers, $n$, increases. More importantly, however, the performance of the P.S.S.P.(n) relative to the A.S.S.P.(n) improves as $n$ increases. The most surprising result, however, is that for large $n$ the


than that of the A.S.S.P.(n). In fact, as $n \rightarrow \infty$ the quotient mentioned above approaches $1-2 a$. This result is discussed further in appendix 3. A similar result noted by Cheng [3] has been dismissed since, unlike the present case, it appeared to apply only for large values of the input signal to noise ratio. An examination of the expressions (25) and (26) shows that, in this present case also, the phenomenon could have been expressed in terms of $a ; 1-2 a$ becomes smaller as $a$ increases. However, the present work is only valid for 


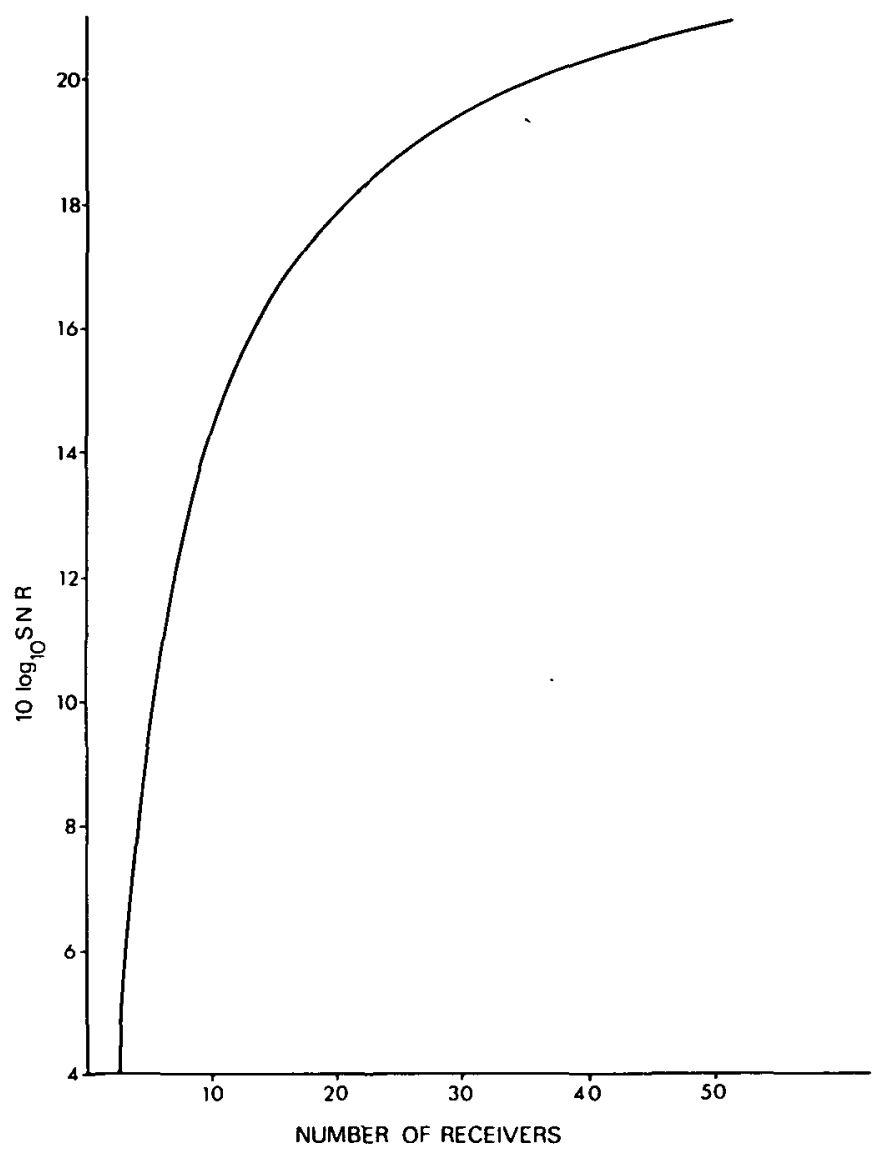

Figure $3-$ Output signal to noise ratio of the polarity sum and square law processor for $T=100$

small $a$, although it is probable that increasing $a$ does have a similar effect to increasing $n$.

Usher [20] also reports a case where the performance of a system with infinite clipping is generally superior on a signal to noise basis. In his work the noise power input was allowed to vary between channels and the above effect was noted especially when this variation was large. This phenomenon may be studied more closely using the techniques of this paper. It may be worth commenting that in cases where one set of receivers has a much higher signal to noise ratio than the others, there may be advantage in only processing the output from the former. Such a procedure would be the extreme case of shaping the input signal and noise before clipping - an obvious extension of the present study. 


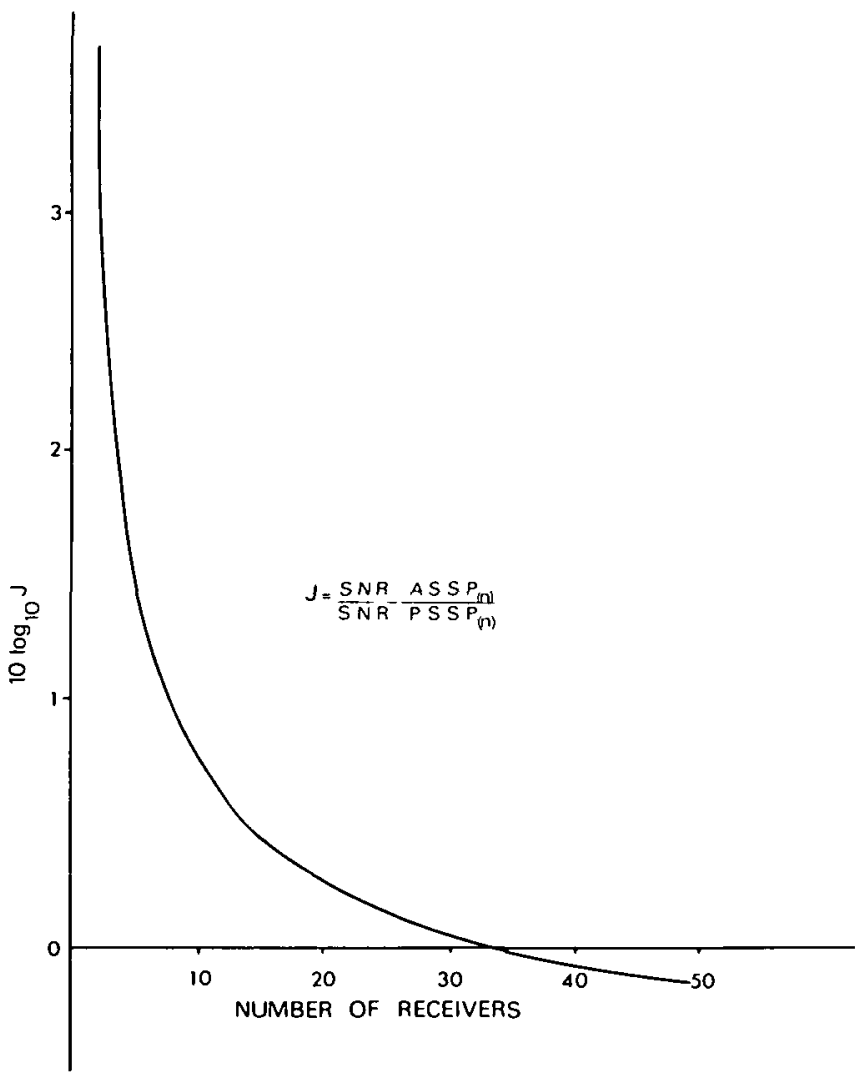

Figure 4-Comparison of output signal to noise ratio of analogue and polarity sum and square law processors for $T=5$

\section{Acknowledgement}

Part of this work was carried out under a grant to the University of Newcastle from the Department of Defence, Australia.

\section{Appendix 1 - partial derivatives of orthant probabilities} needed for the calculation of the required expectations

$$
\begin{aligned}
& \Phi_{1}[a, b, c, d, e]=F\left[a, a b c+d, a b, a b, \frac{a b}{c}+e, a\right] \\
& \Phi_{2}[a, b, c, d, e]=F[a, a b, a b c, a b d+e, a b c d, a c d] \\
& \Phi_{3}[a, b, c, d, e]=F\left[a, a b c, a b d, a b e, \frac{a b d e}{c}, a d e\right]
\end{aligned}
$$


Tables giving the required partial derivatives for $\Phi_{1}(a, b, c, d, e)$ follow; those for $\Phi_{2}(a, b, c, d, e)$ may be found by equating $d$ to zero and replacing the subscript 3 with 4 and 4 with 3 ; those for $\Phi_{3}(a, b, c, d, e)$ follow by equating both $d$ and $e$ to zero.

$$
\begin{gathered}
\Phi_{l}[0, b, c, d, e]=\frac{1}{16 \pi^{2}}(\pi+2 \arcsin d)(\pi+2 \arcsin e) \\
{\left[\frac{\partial \Phi_{1}}{\partial \rho_{l y}}\right]_{a=0}}
\end{gathered}
$$

\begin{tabular}{cc}
\hline$i j$ & Value \\
\hline $12,14,23,34$ & $\frac{1}{8 \pi}$ \\
13 & $\frac{\pi+2 \arcsin e}{8 \pi^{2} \sqrt{1-d^{2}}}$ \\
24 & $\frac{\pi+2 \arcsin d}{8 \pi^{2} \sqrt{1-e^{2}}}$ \\
\hline
\end{tabular}

$$
\left[\frac{\partial^{2} \Phi_{1}}{\partial \rho_{t l} \partial \rho_{k l}}\right]_{a=0}
$$

ijkl Value

$1212,1414,2323,3434$

$$
\frac{d e}{4 \pi^{2} \sqrt{\left(1-d^{2}\right)\left(1-e^{2}\right)}}
$$

1214,2334

$$
\frac{-d}{4 \pi^{2} \sqrt{\left(1-d^{2}\right)\left(1-e^{2}\right)}}
$$

1223,1434

$$
\frac{-e}{4 \pi^{2} \sqrt{\left(1-d^{2}\right)\left(1-e^{2}\right)}}
$$

$1234,1324,1423$

$$
\frac{1}{4 \pi^{2} \sqrt{\left(1-d^{2}\right)\left(1-e^{2}\right)}}
$$

$$
\frac{d(\pi+2 \arcsin e)}{8 \pi^{2}\left(1-d^{2}\right)^{3 / 2}}
$$

$$
\frac{e(\pi+2 \arcsin d)}{8 \pi^{2}\left(1-e^{2}\right)^{3 / 2}}
$$




$$
\left[\frac{\partial^{3} \Phi_{1}}{\partial \rho_{l y} \partial \rho_{k l} \partial \rho_{m n}}\right]_{a=0}
$$

$121212,141414,232323,343434$

$\frac{1}{8 \pi}$

$121213,131414,132323,133434$

$\frac{e}{4 \pi^{2} \sqrt{\left(1-d^{2}\right)^{3}\left(1-e^{2}\right)}}$

$121224,141424,232324,243434$

$$
\frac{d}{4 \pi^{2} \sqrt{\left(1-d^{2}\right)\left(1-e^{2}\right)^{3}}}
$$

121314,132334

$\frac{-1}{4 \pi^{2} \sqrt{\left(1-d^{2}\right)^{3}\left(1-e^{2}\right)}}$

121323,131434

$\frac{-d e}{4 \pi^{2} \sqrt{\left(1-d^{2}\right)^{3}\left(1-e^{2}\right)}}$

$121334,131324,131423$

$\frac{d}{4 \pi^{2} \sqrt{\left(1-d^{2}\right)^{3}\left(1-e^{2}\right)}}$

121424,232434

$$
\frac{-d e}{4 \pi^{2} \sqrt{\left(1-d^{2}\right)\left(1-e^{2}\right)^{3}}}
$$

122324,142434

$$
\frac{-1}{4 \pi^{2} \sqrt{\left(1-d^{2}\right)\left(1-e^{2}\right)^{3}}}
$$

$122434,132424,142324$

$$
\frac{e}{4 \pi^{2} \sqrt{\left(1-d^{2}\right)\left(1-e^{2}\right)^{3}}}
$$

242424

$$
\frac{\left(1+2 e^{2}\right)(\pi+2 \arcsin d)}{8 \pi^{2} \sqrt{\left(1-e^{2}\right)^{5}}}
$$

131313

$$
\frac{\left(1+2 d^{2}\right)(\pi+2 \arcsin e)}{8 \pi^{2} \sqrt{\left(1-d^{2}\right)^{5}}}
$$


Appendix 2 - expectations evaluated using the first three terms in the Taylor series approximation of $\Phi_{1}, \Phi_{2}$ and $\Phi_{3}$

$$
\begin{aligned}
& E\left[A_{i} A_{j} B_{i} B_{l}\right]=\frac{4}{\pi^{2}} \arcsin \left(d_{i}\right) \cdot \arcsin \left(d_{l}\right) \\
& +a_{i 1} \frac{4 b}{\pi^{2}}\left[\frac{c_{11} \cdot \arcsin \left(d_{1}\right)}{\sqrt{1-d_{i}^{2}}}+\frac{\arcsin \left(d_{1}\right)}{c_{j 1} \cdot \sqrt{1-d_{1}^{2}}}\right] \\
& +a_{i j}^{2}\left\{\frac{2 b^{2}}{\pi^{2}}\left[\frac{c_{i t}^{2} d_{i} \cdot \arcsin \left(d_{1}\right)}{\left(1-d_{i}^{2}\right)^{3 / 2}}+\frac{d_{j} \cdot \arcsin \left(d_{i}\right)}{c_{j i}^{2}\left(1-d_{l}^{2}\right)^{3 / 2}}\right]\right. \\
& \left.+\frac{4}{\pi^{2} \sqrt{\left(1-d_{1}^{2}\right)\left(1-d_{l}^{2}\right)}}\left[1+d_{1} d_{1}-2 b d_{1}-2 b d_{1}+2 b^{2}+b^{2} d_{1} d_{1}\right]\right\} \\
& +a_{11}^{3}\left\{\frac{2 b^{3}}{3 \pi^{2}}\left[\frac{c_{l,}^{3}\left(2 d_{1}^{2}+1\right)}{\left(1-d_{1}^{2}\right)^{5 / 2}} \arcsin \left(d_{1}\right)+\frac{\left(2 d_{l}^{2}+1\right)}{c_{\mu(1)}^{3}\left(1-d_{l}^{2}\right)^{5 / 2}} \arcsin \left(d_{1}\right)\right]\right. \\
& +\frac{2 b}{\pi^{2} \sqrt{\left(1-d_{1}^{2}\right)\left(1-d_{1}^{2}\right)}}\left[\frac { c _ { l i } } { ( 1 - d _ { 1 } ^ { 2 } ) } \left(2 d_{i}+2 d_{i}-4 b-4 b d_{1} d_{1}\right.\right. \\
& \left.+3 b^{2} d_{t}+2 b^{2} d_{s}\right) \\
& \left.\left.+\frac{1}{c_{\mu}\left(1-d_{l}^{2}\right)}\left(2 d_{i}+2 d_{\jmath}-4 b-4 b d_{1} d_{1}+2 b^{2} d_{1}+3 b^{2} d_{l}\right)\right]\right\}
\end{aligned}
$$$$
E\left[A, A, B, B_{k}\right]=a_{\imath \jmath} \frac{4 b c_{l k}}{\pi^{2}} \arcsin \left(d_{l}\right)+a_{11}^{2} \frac{4 c_{l k} c_{1 j}}{\pi^{2} \sqrt{1-d_{j}^{2}}}\left[1-2 b d_{l}+2 b^{2}\right]
$$$$
+a_{11}^{3}\left\{\frac{2 b^{3} c_{l k}^{3}}{3 \pi^{2}} \arcsin \left(d_{1}\right)+\frac{2 b c_{l k} c_{i j}^{2}}{\pi^{2}\left(1-d_{f}^{2}\right)^{3 / 2}}\left[2 d_{1}-4 b+3 b^{2} d_{1}\right]\right.
$$$$
\left.+\frac{2 b c_{l k}}{\pi^{2} \sqrt{1-d^{2}}}\left[1+c_{l k}^{2} c_{\imath]}^{2}\right]\left[d,-2 b+b^{2} d_{l}\right]\right\}
$$

$E\left[A, A, B_{k} B_{l}\right]=a_{1 j}^{2} \frac{4 c_{1} c_{i k}}{\pi^{2}}\left[1+2 b^{2}\right]-a_{11}^{3} \frac{4 b^{2} c_{1 j}}{\pi^{2}}\left[c_{l k}\left(1+c_{i k}^{2}\right)+\frac{c_{i k}^{2}}{c_{i k}}\left(1+c_{l l}^{2}\right)\right]$

\section{Appendix 3 - the apparent superiority of the P.S.S.P.}

\section{for large values of $n$.}

The authors are grateful to one of their referees for drawing attention to the apparent paradox inherent in one of the remarks in section 4, viz. "The most surprising result, however, is that for large $n$ the performance of the P.S.S.P. $_{(n)}$ is, using this signal to noise criterion, better than that of the A.S.S.P.(n)". As this referee remarked, under the stated assumptions it has been well documented, e.g. [17], that the A.S.S.P. detector is identical to the 
likelihood ratio detector which is known to be optimal. In view of this paradox it will be useful to discuss this matter in more detail.

Two questions arise:

(1) is the analysis correct?

(2) if so, how is the paradox explained?

A 3.1 The signal to noise ratio of the P.S.S.P. ${ }_{(n)}$ for large $n$

From equation (8), neglecting all powers of $n$ less than the fourth it can be seen that the output signal to noise ratio of the P.S.S.P..$_{(n)}$ is:

$$
\frac{\frac{4}{\pi^{2}}(\arcsin a)^{2}}{\frac{1}{T} \int_{0}^{\infty} \exp \left[\frac{-\mu}{T}\right]\left[E\left[A_{\imath} A_{i} B_{k} B_{l}\right]-\frac{4}{\pi^{2}}(\arcsin a)^{2}\right] d \mu}
$$

Now in this case a closed form expression for $E\left[A_{i} A_{j} B_{k} B_{l}\right]$ can be derived from the work of Cheng [4].

Cheng [4] has shown that the orthant quadrivariate probability correspondir.g to the correlation matrix

$$
\left[\begin{array}{cccc}
1 & a & a b & a b \\
a & 1 & a b & a b \\
a b & a b & 1 & a \\
a b & a b & a & 1
\end{array}\right]
$$

is

$$
\begin{aligned}
& \frac{1}{16}+\frac{1}{4 \pi}[\arcsin (a)+2 \arcsin (a b)] \\
& +\frac{1}{4 \pi^{2}}\left[\{\arcsin (a)\}^{2}-2\{\arcsin (a b)\}^{2}\right] \\
& \quad+\frac{1}{\pi^{2}}\left\{\frac{1}{2} L_{12}\left(-h^{2}\right)+2 L_{12}(h, \arccos a b)-L_{12}\left[h^{2}, \arccos (a)\right]\right\}
\end{aligned}
$$

while Stokes [18] has noted that

$$
E\left[A_{1} A_{1} B_{k} B_{l}\right]=1-16 F(a, a b,-a b, a b,-a b,-a) .
$$

$L_{i 2}$ here denotes the dilogarithm functions of Lewin [15] defined by 


$$
L_{12}(x)=-\int_{0}^{x} \frac{\ln (1-v)}{v} d v
$$

and

$$
L_{12}(x, \theta)=-\frac{1}{2} \int_{0}^{x} \frac{\ln \left(1-2 v \cos \theta+v^{2}\right)}{v} d v
$$

while

$$
h=\frac{1}{2 a b}\left[(1+a)-\left\{(1+a)^{2}-4 a^{2} b^{2}\right\}^{\frac{1}{2}}\right] .
$$

From these two results it can be shown that

$$
\begin{aligned}
& \frac{\pi^{2}}{4} E\left[A_{1} A_{1} B_{k} B_{l}\right]-(\arcsin a)^{2} \\
& \quad=2\left[-(\arcsin a b)^{2}+L_{12}\left(-h^{2}\right)+4 L_{12}(h, \arccos a b)-2 L_{12}\left(h^{2}, \arccos a\right)\right] .
\end{aligned}
$$

A simple series expansion will then give, neglecting powers of $a$ greater than the third, the following expression for the signal to noise ratio of the P.S.S.P.(n) as

$$
\begin{aligned}
& \frac{a^{2}}{\frac{1}{T} \int_{0}^{\infty} 2 a^{2} b^{2}(1-2 a) \exp \left(-\frac{\mu}{T}\right) d \mu} \\
& \quad=\frac{1}{2 K(1-2 a)} .
\end{aligned}
$$

This alternative approach confirms this aspect of the analysis of the main text so the paradox remains.

\section{A 3.2 The paradox}

The authors were careful to include the words 'using this signal to noise criterion'* in the sentence, quoted from the text, at the beginning of this appendix. Clearly different criteria lead to different conclusions and, in particular, the likelihood ratio detector employs a different criterion which involves not only the signal to noise ratio in the presence of signal but also the noise power in the absence of signal.

An examination of equations (25) and (26) will show that, in the absence of signal, the noise variance of the P.S.S.P.(n) is larger than that of the

* These words were not italicized in the paper as originally submitted. 
A.S.S.P.(n). Accordingly, the probability of false alarm in the case of the P.S.S.P. ${ }_{(n)}$ is greater than that of the A.S.S.P. ${ }_{(n)}$ or, alternatively, if the P.S.S.P.(n) is adjusted to give the same probability of false alarm as the A.S.S.P.(n) then its apparent superior probability of detection will no doubt disappear. One of us (Keats) has pursued this matter in more detail and it may form the basis of a future paper.

\section{References}

[1] T. G. Birdsall and G. T. Kaye, 'SCAR - the MPT scattering array', Proceedings of Conference on Signal Processing for Arrays held at the Weapons Research Establishment, Salisbury, South Australia, 1975.

[2] M. C. Cheng, 'On a class of non-linear transformations of Gaussian random processes', Ph.D. Thesis, University of Adelaide (1968).

[3] M. C. Cheng, 'The clipping loss in correlation detectors for arbitrary input signal to noise ratios', I.E.E.E. Trans. on Information Theory, IT-14 (1968), 382-389.

[4] M. C. Cheng, 'The orthant probabilities of four Gaussian variates', Ann. Math. Stat., 40 (1969), 152-161.

[5] D. J. Edelblute, J. M. Fisk and G. L. Kinnison, 'Criteria for optimum signal detection for arrays', J. Acoust. Soc. Amer., 41 (1967) 199-205

[6] H. Ekre, 'Polarity coincidence correlation detection of a weak noise source', I.E.E.E. Trans. on Information Theory, IT-9 (1963), 18-23.

[7] J. J. Faran, Jr. and R. Hills, Jr., The Application of Correlation Techniques to Acoustic Receiving Systems, (Technical Memo No. 28, Acoust. Res. Lab., Harvard, 1952).

[8] C. W. Helstrom, Statistical Theory of Signal Detection, (London, Pergamon Press, 2nd ed., 1960).

[9] T. Kailath, 'A general likelihood formula for random signals in Gaussian noise', I.E.E.E. Trans. on Information Theory, IT-15 (1969) 350-361.

[10] T. Kailath, 'The innovations approach to detection and estimation theory', Proc. I.E.E.E. 58 (1970), 680-695.

[11] T. Kailath, 'Some extensions of the innovations theorem', B.S.T.J. 50 (1971), 1487-1494.

[12] M. Kanefsky, 'Detection of weak signals with polarity coincidence arrays', I.E.E.E. Trans. on Information Theory IT-12 (1966), 260-268.

[13] R. G. Keats, 'Sum and square law signal processors with multiple clipped inputs', Proceedings of Conference on Signal Processing for Arrays held at Weapons Research Establishment, Salisbury, South Australia, 1975.

[14] J. H. Laning and R. H. Battin, Random Processes in Automatic Control, (McGraw-Hill, 1956).

[15] L. Lewin, Dilogarithms and Associated Functions (London, MacDonald Press, 1958).

[16] R. L. Plackett, 'A reduction formula for normal multivariate integrals', Biometrika 41 (1954), 351-360.

[17] P. M. Schultheiss and F. B. Tuteur, 'Optimum and suboptimum detection of directional Gaussian signals in an isotropic Gaussian noise field, Part II: degradation of detectability due to clipping', I.E.E.E. Trans. on Military Electronics MIL-9 (1965), 208-211. 
[18] B. J. Stokes, 'Signal detection using infinite clipping: A survey and an application', M. Math. Thesis, University of Newcastle (1973).

[19] J. B. Thomas and T. R. Williams, 'On the detection of signals in nonstationary noise by product arrays', Journal of Acoust. Soc. of America 31 (1959), 453-462.

[20] T. Usher, Jr., 'Signal detection by arrays in noise fields with local variations', Journal of Acoust. Soc. of America 36, No. 8 (1964), 1444-1449.

[21] H. L. Van Trees, Detection, Estimation and Modulation Theory - Part III: Radar-Sonar Signal Processing and Gaussian Signals in Noise, (New York, Wiley, 1971).

Department of Mathematics,

University of Newcastle,

Newcastle,

Australia. 